\title{
Computational sound field in a virtual environment via field data in an arbitrary real environment
}

\author{
Xiaolei $\mathrm{Li}^{*}$, Dazhi Gao, and Ning Wang \\ Department of Marine Technology, Ocean University of China, Qingdao 266100, China
}

\begin{abstract}
It is useful to compute sound field of a source in a virtual environment which is different from the measurement environment. For example, some properties of sound source, such as directivity index and frequency response curve, are required to be measured in an anechoic room or free space, but both of them cannot be always accessible. Consequently, it will be useful to compute sound field of a source in free space when sound field of the source is not measured in the free space. In the aforementioned example, the free space is a virtual environment. Based on reciprocity theorem and modal expansion, a method to predict sound field of a source in a virtual environment is given in this paper when the scattering effect of the source can be neglected. Reciprocity theorem builds the relationship between measured sound field and predicted sound field, which plays an important role in the method. Green's function in the virtual environment is needed in the method. To restrict measurement points on an enclosed surface, the Green's function is expanded by a set of modes. A simulation is given to examine the validity of the method.
\end{abstract}

\section{Introduction}

Virtual acoustic reconstruction (VAR) which mainly focuses on computer-aided simulation of building acoustics is a well-established tool for the study of the acoustic behavior of such spaces and is often combined with virtual acoustic representation (auralisation) to provide direct aural impression of their response to speech or music signals [1-4]. To compute sound field stimulated by a source in a virtual environment by VAR, the properties of the source, such as directivity and frequency response, must be known. However the properties of a sound source cannot be measured directly in an ordinary room, and the sound field stimulated by the source with unknown properties in a virtual environment cannot computed by VAR. When the virtual environment is equal to the real environment, sound field reconstruction methods, such as near-field acoustical holography (NAH) [5-16], inverse boundary element method (IBEM) [17-19], and least-squares method (LSM) [20, 21], can be used to compute sound field stimulated by a certain source and the properties of the source do not need to be known in advance. However, when a virtual environment is different from the real environment, sound field reconstruction is not a suitable method to compute sound field stimulated by a certain source in a virtual environment.

In this paper, a method called extended virtual acoustic reconstruction (EVAR) is proposed which maps sound field in an arbitrary real environment to that in a virtual environment without knowing properties of the excitation source in advance when scattering effect of the source can be neglected. EVAR is derived based mainly on reciprocity theorem $[22,23]$. Use SFR and $\mathrm{SFV}$ as the acronyms for sound field in the real environment and that in a virtual environment respectively. To obtain SFV, the Green's function in the virtual environment should be known. However one cannot reconstruct SFV with the Green's function directly based on reciprocity theorem when SFR is not accessible in the whole domain. This problem can be overcome by expanding the Green's function with a set of modes which satisfy homogeneous Helmholtz equation, and the measurement points are restricted on an enclosed surface which contains the sound source. Both pressure and its gradient need to be measured on the measurement surface in EVAR. A simulation is given to examine the validity of this method.

This paper is organized as follows. Section II presents the EVAR using reciprocity theorem, which is examined by a simulation in section III. A conclusion is given in section IV.

\section{Theory of EVAR}

In this paper, the sound field is considered in frequency domain which is related to time domain by Fourier transform. Let $\varphi_{1}(\mathbf{r})$ be the SFR which satisfies both the boundary conditions in the real environment and inhomogeneous Helmholtz equation

$$
\nabla^{2} \varphi_{1}(\mathbf{r})+k^{2} \varphi_{1}(\mathbf{r})=S(\mathbf{r})
$$

where $k=\omega / c$ is wave number, $\omega$ is angular frequency, $c$ is sound speed of medium and $S(\mathbf{r})$ is source term

* Corresponding author: $1 \mathrm{xl}$ ouc@163.com 
whose support set is limited in a finite domain $D$, see Fig.1.

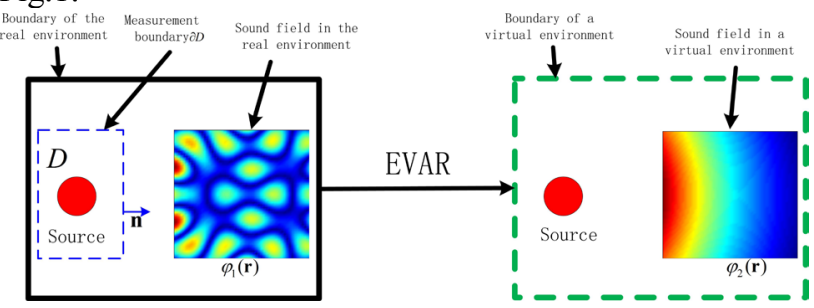

Fig. 1. (Color online) Sketch map of the EVAR method. A source $S(\boldsymbol{r})$ locates in the domain $D$, the boundary surface of $D$ is denoted by $\partial D$ and the normal vector $\mathbf{n}$ on $\partial D$ is directed away from $D$. Measurement points are distributed on the $\partial D$. Both sound field and its normal derivative are measured on the $\partial D$. The sound field $\varphi_{2}(\mathbf{r})$ which is stimulated by the same source $S(\mathbf{r})$ in a virtual environment can be predicted by $\varphi_{1}(\mathbf{r})$ which is the sound field in the real environment and its derivative on the $\partial D$ according to EVAR.

Fig.1 Let $\varphi_{2}(\mathbf{r})$ be the SFV which satisfies the same equation as $\varphi_{1}(\mathbf{r})$ but the boundary conditions in a virtual environment.

$$
\nabla^{2} \varphi_{2}(\mathbf{r})+k^{2} \varphi_{2}(\mathbf{r})=S(\mathbf{r})
$$

It is well known that $\varphi_{2}(\mathbf{r})$ can be represented by the Green's function $G_{t}\left(\mathbf{r}, \mathbf{r}_{0}\right)$ in the virtual environment

$$
\varphi_{2}(\mathbf{r})=\int_{D} G_{t}\left(\mathbf{r}, \mathbf{r}_{0}\right) S\left(\mathbf{r}_{0}\right) d \mathbf{r}_{0}
$$

The integral in Eq. (3) can be obtained by the reciprocity relationship between $\varphi_{1}(\mathbf{r})$ and $G_{t}\left(\mathbf{r}, \mathbf{r}_{0}\right)$ when $\varphi_{1}(\mathbf{r})$ is accessible in the whole domain $D$ as Eq. (4) shows.

$$
\begin{aligned}
& \oint_{\partial D}\left[G_{t}\left(\mathbf{r}, \mathbf{r}_{0}\right) \nabla_{\mathbf{r}_{0}} \varphi_{1}\left(\mathbf{r}_{0}\right)-\varphi_{1}\left(\mathbf{r}_{0}\right) \nabla_{\mathbf{r}_{0}} G_{t}\left(\mathbf{r}, \mathbf{r}_{0}\right)\right] \cdot \mathbf{n} d S+\varphi_{1}(\mathbf{r}) \\
& =\int_{D} G_{t}\left(\mathbf{r}, \mathbf{r}_{0}\right) S\left(\mathbf{r}_{0}\right) d \mathbf{r}_{0}
\end{aligned}
$$

where $\nabla_{\mathbf{r}_{0}}$ is gradient with respect to $\mathbf{r}_{0}$ and $\mathbf{n}$ is the unit normal vector on $\partial D$ directed away from $D$, see figure 1 . However it is difficult to obtain $\varphi_{1}(\mathbf{r})$ in the whole domain $D$. To tackle this problem, the following trick is adopted.

Define a set of functions $\left\{u_{\alpha}(\mathbf{r})\right\}$ which are complete, linearly independent and satisfy the homogeneous Helmholtz equation, see Eq. (5). The parameter $\alpha$ in $u_{\alpha}(\mathbf{r})$ consists of a discrete part $D(\alpha)$ and a continuous part $C(\alpha)$.

$$
\nabla^{2} u_{\alpha}(\mathbf{r})+k^{2} u_{\alpha}(\mathbf{r})=0
$$

Here "complete" means any sound field $\varphi(\mathbf{r})$ which satisfies homogeneous Helmholtz equation in a domain $\Omega$ can be expanded by $u_{\alpha}(\mathbf{r})$ in $\Omega$

$$
\varphi(\mathbf{r})=\int_{C(\alpha)} \kappa(\alpha) u_{\alpha}(\mathbf{r}) d \alpha+\sum_{D(\alpha)} v(\alpha) u_{\alpha}(\mathbf{r}) \quad \mathbf{r} \in \Omega
$$

where $\kappa(\alpha)$ and $v(\alpha)$ are independent of $\mathbf{r}$. Then Green's function $G_{t}\left(\mathbf{r}, \mathbf{r}_{0}\right)$ can be expanded by $u_{\alpha}(\mathbf{r})$ in a domain $\Omega^{\prime}$ where $\mathbf{r}_{0} \notin \Omega^{\prime}$.

$G_{t}\left(\mathbf{r}, \mathbf{r}_{0}\right)=\int_{C(\alpha)} \kappa_{f}\left(\alpha, \mathbf{r}_{0}\right) u_{\alpha}(\mathbf{r}) d \alpha+\sum_{D(\alpha)} v_{g}\left(\alpha, \mathbf{r}_{0}\right) u_{\alpha}(\mathbf{r}) \quad \mathbf{r} \in \Omega^{\prime}$

when $\mathbf{r} \in \Omega^{\prime}$,

$$
\nabla_{\mathbf{r}_{0}}^{2} G_{t}\left(\mathbf{r}, \mathbf{r}_{0}\right)+k^{2} G_{t}\left(\mathbf{r}, \mathbf{r}_{0}\right)=0
$$

According to Eq. (6) and Eq. (7), both $\kappa_{f}\left(\alpha, \mathbf{r}_{0}\right)$ and $v_{g}\left(\alpha, \mathbf{r}_{0}\right)$ satisfy homogeneous Helmholtz equation.

$$
\begin{aligned}
& \nabla_{\mathbf{r}_{0}}^{2} \kappa_{f}\left(\alpha, \mathbf{r}_{0}\right)+k^{2} \kappa_{f}\left(\alpha, \mathbf{r}_{0}\right)=0 \\
& \nabla_{\mathbf{r}_{0}}^{2} v_{g}\left(\alpha, \mathbf{r}_{0}\right)+k^{2} v_{g}\left(\alpha, \mathbf{r}_{0}\right)=0
\end{aligned}
$$

Suppose Green's function $G_{t}\left(\mathbf{r}, \mathbf{r}_{0}\right)$ can be expanded by $u_{\alpha}$ (r) uniformly when $\mathbf{r}_{0}$ belongs to the support set of $S\left(\mathbf{r}_{0}\right)$ and $\mathbf{r} \in \Omega^{\prime}$. Then combining Eq. (3) and Eq. (6) at first, one can obtain

$$
\begin{aligned}
\varphi_{2}(\mathbf{r})= & \int_{D} S\left(\mathbf{r}_{0}\right) \int_{C(\alpha)} \kappa_{f}\left(\alpha, \mathbf{r}_{0}\right) u_{\alpha}(\mathbf{r}) d \alpha d \mathbf{r}_{0}+ \\
& \int_{D} S\left(\mathbf{r}_{0}\right) \sum_{D(\alpha)} v_{g}\left(\alpha, \mathbf{r}_{0}\right) u_{\alpha}(\mathbf{r}) d \mathbf{r}_{0} \\
= & \int_{C(\alpha)} u_{\alpha}(\mathbf{r}) \int_{D} S\left(\mathbf{r}_{0}\right) \kappa_{f}\left(\alpha, \mathbf{r}_{0}\right) d \mathbf{r}_{0} d \alpha+ \\
& \sum_{D(\alpha)} u_{\alpha}(\mathbf{r}) \int_{D} S\left(\mathbf{r}_{0}\right) v_{g}\left(\alpha, \mathbf{r}_{0}\right) d \mathbf{r}_{0}
\end{aligned}
$$

where $\mathbf{r} \in \Omega^{\prime}$. Note that $\kappa_{f}\left(\alpha, \mathbf{r}_{0}\right), v_{g}\left(\alpha, \mathbf{r}_{0}\right)$ and $u_{\alpha}(\mathbf{r})$ can be obtained by analytic method or numerical method under certain boundary conditions, and to obtain $\varphi_{2}(\mathbf{r})$, only the integral about $\mathbf{r}_{0}$ in Eq. (9) needs to be known. Actually, both $\int_{D} S\left(\mathbf{r}_{0}\right) \kappa_{f}\left(\alpha, \mathbf{r}_{0}\right) d \mathbf{r}_{0}$ and $\int_{D} S\left(\mathbf{r}_{0}\right) v_{g}\left(\alpha, \mathbf{r}_{0}\right) d \mathbf{r}_{0}$ can be obtained by $\varphi\left(\mathbf{r}_{0}\right)$ and its normal derivative on $\partial D$ based on the reciprocity theorem. According to Eq. (1), Eq. (8) and reciprocity theorem, below equations can be derived.

$$
\begin{aligned}
& \oint_{\partial D}\left[\kappa_{f}\left(\alpha, \mathbf{r}_{0}\right) \nabla_{\mathbf{r}_{0}} \varphi_{1}\left(\mathbf{r}_{0}\right)-\varphi_{1}\left(\mathbf{r}_{0}\right) \nabla_{\mathbf{r}_{0}} \kappa_{f}\left(\alpha, \mathbf{r}_{0}\right)\right] \cdot \mathbf{n} d S \\
& =\int_{D} \kappa_{f}\left(\alpha, \mathbf{r}_{0}\right) S\left(\mathbf{r}_{0}\right) d \mathbf{r}_{0} \\
& \oint_{\partial D}\left[v_{g}\left(\alpha, \mathbf{r}_{0}\right) \nabla_{\mathbf{r}_{0}} \varphi_{1}\left(\mathbf{r}_{0}\right)-\varphi_{1}\left(\mathbf{r}_{0}\right) \nabla_{\mathbf{r}_{0}} v_{g}\left(\alpha, \mathbf{r}_{0}\right)\right] \cdot \mathbf{n} d S \\
& =\int_{D} v_{g}\left(\alpha, \mathbf{r}_{0}\right) S\left(\mathbf{r}_{0}\right) d \mathbf{r}_{0}
\end{aligned}
$$

Define

$$
\left\{\varphi\left(\mathbf{r}_{0}\right), \varphi_{1}\left(\mathbf{r}_{0}\right)\right\}=\oint_{\partial D}\left[\varphi\left(\mathbf{r}_{0}\right) \nabla_{\mathbf{r}_{0}} \varphi_{1}\left(\mathbf{r}_{0}\right)-\varphi_{1}\left(\mathbf{r}_{0}\right) \nabla_{\mathbf{r}_{0}} \varphi\left(\mathbf{r}_{0}\right)\right] \cdot \mathbf{n} d S
$$

Combining with Eq. (10) and Eq. (11), Eq. (9) can be rewritten as

$$
\begin{aligned}
\varphi_{2}(\mathbf{r})= & \int_{C(\alpha)}\left\{\kappa_{f}\left(\alpha, \mathbf{r}_{0}\right), \varphi_{1}\left(\mathbf{r}_{0}\right)\right\} u_{\alpha}(\mathbf{r}) d \alpha \\
& +\sum_{D(\alpha)}\left\{v_{g}\left(\alpha, \mathbf{r}_{0}\right), \varphi_{1}\left(\mathbf{r}_{0}\right)\right\} u_{\alpha}(\mathbf{r})
\end{aligned}
$$


where $\mathbf{r} \in \Omega^{\prime}$. From Eq. (12) one can find that the SFV $\varphi_{2}(\mathbf{r})$ can be represented by the SFR $\varphi_{1}(\mathbf{r})$. Actually, Eq. (12) builds a mapping between two sound fields satisfying different boundary conditions and is the central part of EVAR. In the next section, a simulation is given to examine EVAR.

\section{Simulations}

To illuminate EVAR more clearly and to examine its validity, a simulation is given in this part and the simulation is restricted in the two-dimensional space. Supposing there are six identical point sound sources whose working frequency is $f=1500 \mathrm{~Hz}$ in a rectangle cavity. The medium in the cavity is homogeneous and isotropic with sound speed $c=1500 \mathrm{~m} / \mathrm{s}$. Length of the rectangle cavity is $a=6.3 \lambda$ and width is $b=5.3 \lambda$ where $\lambda=c / f=1 \mathrm{~m}$ is the sound wavelength in the medium. Acoustic boundary conditions of the rectangle cavity are perfectly rigid. The Cartesian coordinate system is shown in Fig.2(a). Then the coordinates of the sound sources are $(3.65 \lambda, 2.65 \lambda),(2.90 \lambda, 3.08 \lambda)$, $(2.90 \lambda, 2.22 \lambda),(3.30 \lambda, 2.65 \lambda),(3.08 \lambda, 2.78 \lambda)$ and $(3.08 \lambda, 2.52 \lambda)$, respectively. Fig.2(b) gives the distribution of the absolute value of $\operatorname{SFR} \varphi_{1}(\mathbf{r})$ in the cavity. The measurement surface $\partial D$ is consistent with the rigid boundary of the rectangle cavity.

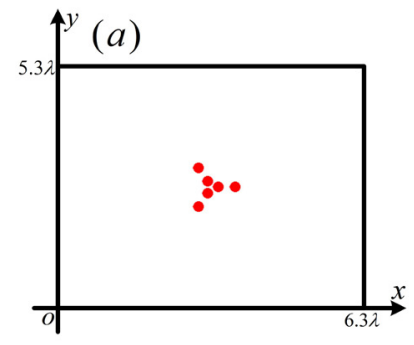

(b)

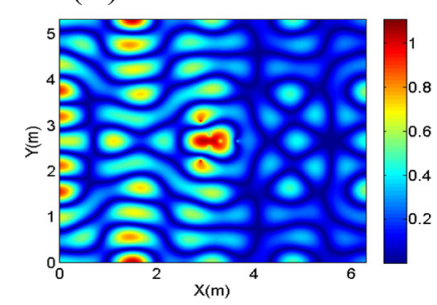

Fig. 2. (Color online) (a) The real environment is a rectangle cavity which has perfectly rigid boundaries and six identical point sound sources distributed in the rectangle cavity. The coordinates of the sound sources are $(3.65 \lambda, 2.65 \lambda)$, $(2.90 \lambda, 3.08 \lambda),(2.90 \lambda, 2.22 \lambda),(3.30 \lambda, 2.65 \lambda)$, $(3.08 \lambda, 2.78 \lambda)$ and $(3.08 \lambda, 2.52 \lambda)$, respectively. (b) Absolute value of the $\operatorname{SFR} \varphi_{1}(\mathbf{r})$ in the rectangle cavity.

Place the same point sources in a two-dimensional ideal waveguide with $H$ height and build the same Cartesian coordinate system as figure 2(a), see Figure $3(\mathrm{a})$. SFV $\varphi_{2}(\mathbf{r})$ should satisfy the following boundary conditions in the two-dimensional ideal waveguide case.

$$
\left\{\begin{array}{l}
\left.\varphi_{2}(\mathbf{r})\right|_{y=0}=0 \\
\left.\frac{\partial \varphi_{2}(\mathbf{r})}{\partial y}\right|_{y=H}=0
\end{array}\right.
$$

where $\mathbf{r}=(x, y)$. The Green's function in a twodimensional ideal waveguide has the following form [24]

$G_{I}\left(\mathbf{r}, \mathbf{r}_{0}\right)=-\frac{i}{H} \sum_{m=1}^{\infty}\left[\sin \left(k_{y m} y_{0}\right) \sin \left(k_{y m} y\right) \times \frac{\exp \left(i k_{x m}\left|x-x_{0}\right|\right)}{k_{x m}}\right]$

where $\mathbf{r}_{0}=\left(x_{0}, y_{0}\right), k_{y m}=(m-1 / 2) \pi / H, k_{x m}^{2}+k_{y m}^{2}=k^{2}$, $\operatorname{Re}\left(k_{x m}\right) \geq 0$ and $\operatorname{Im}\left(k_{x m}\right) \geq 0$. In the ideal waveguide case, evanescent waves are also not considered, i.e. $\operatorname{Im}\left(k_{x m}\right)=0$, when using EVAR. Then Eq. (14) can be rewritten as below (only $x>x_{0}$ is considered).

$$
\begin{aligned}
G_{I}\left(\mathbf{r}, \mathbf{r}_{0}\right) \approx-\frac{i}{H} \sum_{m=1}^{M} & {\left[\frac{1}{k_{x m}} \sin \left(k_{y m} y_{0}\right) \exp \left(-i k_{x m} x_{0}\right) \times\right.} \\
& \left.\sin \left(k_{y m} y\right) \times \exp \left(i k_{x m} x\right)\right]
\end{aligned}
$$

where $M$ satisfies $k_{y M} \leq k<k_{y(M+1)}$,

$$
k_{y m}=(-m+1 / 2) \pi / H
$$

for $m<0$ and $k_{x m}=-\sqrt{k^{2}-k_{y m}^{2}}$ for $m<0$ Compared with Eq. (6), one can get $G_{t}\left(\mathbf{r}, \mathbf{r}_{0}\right)=G_{I}\left(\mathbf{r}, \mathbf{r}_{0}\right), \alpha=m$, $C(m)=\varnothing$ ( $\varnothing$ represents the empty set) $D(m)=$ $\left\{D_{1}(m), D_{2}(m)\right\}, D_{1}(m)=\{1,2, \cdots, M\}, D_{2}(m)=\{-1,-2$, $\cdots,-M\}, u_{m}(\mathbf{r})=\sin \left(k_{y m} y\right) \exp \left(i k_{x m} x\right)$, and

$$
v_{g}\left(m, \mathbf{r}_{0}\right)=-i u_{m}^{*}\left(\mathbf{r}_{0}\right) /\left.\left(H k_{x m}\right)\right|_{m \in D_{1}(m)}
$$
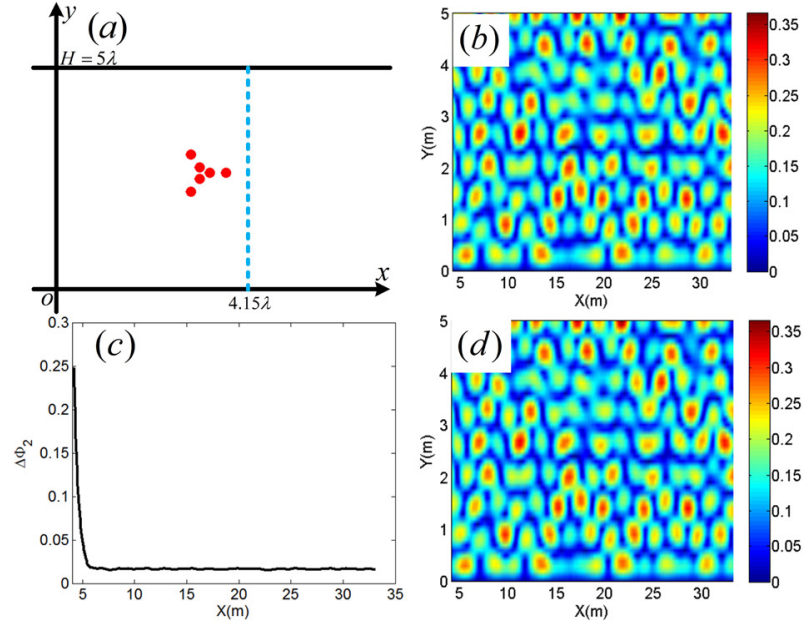

Fig. 3. (Color online) (a) Six identical point sound sources distributed in the two-dimensional ideal waveguide. The coordinates of the sound sources are $(3.65 \lambda, 2.65 \lambda)$,

$(2.90 \lambda, 3.08 \lambda),(2.90 \lambda, 2.22 \lambda),(3.30 \lambda, 2.65 \lambda)$,

$(3.08 \lambda, 2.78 \lambda)$ and $(3.08 \lambda, 2.52 \lambda)$ respectively. SFV is computed in the domain $\Omega^{\prime}=\{\mathbf{r} \mid x \geq 4.15 \lambda\}$. (b) Absolute value of the accurate SFV $\varphi_{2}^{a}(\mathbf{r})$ in the $\Omega^{\prime}$. (c) Error of the predicted SFV $\varphi_{2}(\mathbf{r})$ in the ideal waveguide case. (d) Absolute value of the predicted $\operatorname{SFV} \varphi_{2}(\mathbf{r})$ in the $\Omega$ ' according to EVAR.

Let $H$ equals $5 \lambda$ and only concerns the domain $\Omega^{\prime}=\{\mathbf{r} \mid x \geq 4.15 \lambda\}$ in the ideal waveguide simulation, see Figure 3(a). Note that $G_{I}\left(\mathbf{r}, \mathbf{r}_{0}\right)$ can be expanded by $u_{m}(\mathbf{r})$ uniformly in $\Omega^{\prime}$ for different point sources, then EVAR can be used to reconstruct SFV $\varphi_{2}(\mathbf{r})$ by SFR 
$\varphi_{1}(\mathbf{r})$, and $\left|\varphi_{1}(\mathbf{r})\right|$ is shown in Figure 3(d). The integrals in Eq. (11) are computed by trapezoidal rule [25] with step size $\Delta S=0.05 \lambda$ when reconstructing $\operatorname{SFV} \varphi_{2}(\mathbf{r})$ by EVAR. To determine the error of $\varphi_{2}(\mathbf{r})$, the accurate SFV $\varphi_{2}^{a}(\mathbf{r})$ is required. $\varphi_{2}^{a}(\mathbf{r})$ is computed by combining Eq. (3) and Eq. (14) with $\infty$ being replaced by $N$ which satisfies $k_{y(N-1)} \leq 3 k<k_{y N} \cdot\left|\varphi_{2}^{a}(\mathbf{r})\right|$ is shown in Figure 3(b). Define the reconstruction error of $\varphi_{2}(\mathbf{r})$ as a function of $x$ :

$$
\Delta \Phi_{2}(x)=\frac{\int_{0}^{H}\left|\varphi_{2}(\mathbf{r})-\varphi_{2}^{a}(\mathbf{r})\right|_{\mathbf{r}=(x, y)} d y}{\int_{0}^{H}\left|\varphi_{2}(\mathbf{r})\right|_{\mathbf{r}=(4.15 \lambda, y)} d y}
$$

which is plotted in Fig.3(c). As Fig.3(c) shows, $\Delta \Phi_{2}(x)$ decreases along $x$ because the influence of evanescent waves decreases along $x$. Through this simulation, the validity of EVAR is verified.

\section{Conclusions}

A method called EVAR is proposed in this paper, which can reconstruct SFV by SFR. To make sure that SFR $\varphi_{1}(\mathbf{r})$ and its gradient are only measured on an enclosed surface $\partial D$ in the method, the Green's function $G_{t}\left(\mathbf{r}, \mathbf{r}_{0}\right)$ in the virtual environment is expanded by a set of complete functions $\left\{u_{\alpha}(\mathbf{r})\right\}$ which satisfy homogeneous Helmholtz equation when applying EVAR. A simulation is given to verify the validity of EVAR. Because there are not any requirements on the boundary conditions in the real environment and in the virtual environment in the EVAR, the method proposed in this paper maybe useful in dereverberation, home theatre, virtual reality, sound source calibration etc.

\section{References}

1. G. Iannace, U. Berardi, Proc. Mtgs. Acoust 30, 015011 (2017)

2. F. Martellotta, L. A. Morales, Proceedings of forum acusticum (2014)

3. U. Berardi, G. Iannace, L. Maffei, Journal of Cultural Heritage 19, 555 (2016)

4. S. L. Vassilantonopoulos, J. M. Mourjopoulos, acta acustica · ACUSTICA 87, 604 (2001)

5. S. F. Wu, J. Acoust. Soc. Am. 124, 2680 (2008)

6. E. G. Williams, J. D. Maynard, Phys. Rev. Lett. 45, 557 (1980)

7. E. G. Williams, J. D. Maynard, E. Skudrzyk, J. Acoust. Soc. Am. 68, 340 (1980)

8. E. G. Williams, H. D. Dardy, R. G. Fink, J. Acoust. Soc. Am. 78, 789 (1985)

9. J. D. Maynard, E. G. Williams, Y. Lee, J. Acoust. Soc. Am. 78, 1395 (1985)

10. S. I. Hayek, T. W. Luce, J. Vib., Acoust., Stress, and Reliab 110, 91 (1988)

11. E. G. Williams, B. H. Houston, P. C. Herdic, J. Acoust. Soc. Am 114, 1322 (2003)
12. A. Sarkissian, J. Acoust. Soc. Am 115, 1593 (2004)

13. A. Sarkissian, J. Acoust. Soc. Am 118, 671 (2005)

14. M. Lee, J. S. Bolton, J. Acoust. Soc. Am 118, 3721 (2005)

15. K. Saijyou, S. Yoshikawa, J. Acoust. Soc. Am 110, 2007 (2001)

16. A. M. Pasqual, Mechanical Systems and Signal Processing, 90, 285 (2017)

17. B. K. Gardner, R. J. Bernhard, J. Vib., Acoust., Stress, and Reliab 110, 84 (1988)

18. W. A. Veronesi, J. D. Maynard, J. Acoust. Soc. Am. 85, 588 (1989)

19. A. N. Norris, J. Acoust. Soc. Am. 102, 2098 (1997)

20. Z. Wang, S. F. Wu, J. Acoust. Soc. Am. 102, 2020 (1997)

21. Y. Wang, K. Chen, J. Acoust. Soc. Am. 142, 1858 (2017)

22. J. Lin, X. Li, N. Wang, Chinese Physics B 25, 124303 (2016)

23. X. Li, G. Yu, N. Wang, D. Gao, H. Wang, J. Acoust. Soc. Am. 141, EL1 (2017)

24. F. B. Jensen, W. A. Kuperman, M. B. Porter, H. Schmidt, Computional Ocean Acoustics (Second Edition) (Springer, 2011) 344-349

25. T. Sauer, Numerical Analysis (Pearson Education, 2012) 254-257 